\title{
Energy Velocity of Diffusing Waves in Strongly Scattering Media
}

\author{
H. P. Schriemer, ${ }^{1}$ M. L. Cowan, ${ }^{1}$ J. H. Page,${ }^{1}$ Ping Sheng, ${ }^{2}$ Zhengyou Liu, ${ }^{2}$ and D. A. Weitz ${ }^{3}$ \\ ${ }^{1}$ Department of Physics, University of Manitoba, Winnipeg, Manitoba, Canada R3T 2N2 \\ ${ }^{2}$ Department of Physics, The Hong Kong University of Science and Technology, Clear Water Bay, Kowloon, Hong Kong \\ ${ }^{3}$ Department of Physics and Astronomy, University of Pennsylvania, Philadelphia, Pennsylvania 19104-6396
}

(Received 9 June 1997)

\begin{abstract}
Measurements of the diffusive transport of multiply scattered ultrasonic waves show that the energy velocity is very similar in magnitude and frequency dependence to the group velocity. Our data are accurately described using a theoretical model that accounts for the renormalization of scattering by the coupling between neighboring scatterers, quantitatively predicting the scattering delay that causes the strong frequency dependence of these velocities seen in our experiments. This gives a unified physical picture of the velocities of energy transport by both diffusive and ballistic waves. [S0031-9007(97)04300-7]
\end{abstract}

PACS numbers: 43.35. $+\mathrm{d}, 43.20 .+\mathrm{g}, 62.30 .+\mathrm{d}$

In recent years, there has been a tremendous revival of interest in the propagation of classical waves through inhomogeneous media containing random scatterers [1,2]. When the scattering is strong, the propagation is typically very well described using the diffusion approximation, and this has successfully facilitated the interpretation of a wide range of fascinating wave phenomena. Even though the transport is diffusive, it is, nevertheless, still essential to define several propagation velocities. These include the group $\left(v_{g}\right)$ and phase $\left(v_{p}\right)$ velocities which characterize the ballistic, or unscattered, component of the incident waves. For diffusive waves, the relevant velocity is the energy velocity $v_{e}$ which is defined as the ratio of the energy flux to the energy density [3], and which corresponds to the average local velocity of energy transport in the diffusion process, since $v_{e}$ is related to the wave diffusion coefficient $D$ by $D=v_{e} l^{*} / 3$. Here $l^{*}$ is the transport mean free path, or the distance the waves must propagate until their direction is randomized. Within this scenario, the energy velocity represents a distinctly different type of velocity, as wave coherence is no longer relevant. The first calculations for diffusive waves by the Amsterdam group [4], carried out for low volume fractions of scatterers $\phi$, correctly accounted for the extremely low value of $\boldsymbol{v}_{e}$ found in light scattering experiments; they also predicted a strong frequency dependence for $v_{e}$ in the vicinity of Mie resonances as a result of the temporary storage of wave energy inside the scatterer [4-7]. However, these calculations fail as $\phi$ is increased. Following an idea proposed by Sheng [2], recent Coherent Potential Approximation (CPA) calculations $[8,9]$ have attempted to rectify this deficiency for higher volume fractions, and have suggested that the large variations of $v_{e}$ with frequency are washed out with increasing $\phi$; these results are purported to be in excellent agreement $[8,9]$ with the limited experimental data that currently exist for $v_{e}[10,11]$. However, in the intermediate frequency regime where resonant scattering occurs, such CPA theories become suspect [12], raising questions about their reliability. In addition to these ques- tions about the behavior of $v_{e}$, there have been conflicting ideas about the possible connection between the energy velocity of diffusive waves and the group velocity, which normally describes the coherent transport of energy by ballistic wave pulses. For example, the Amsterdam group [4] predicted that $v_{e}$ is entirely different from the "strongly anomalous" group velocity, while a more recent calculation for diffusive scalar waves has suggested that $v_{e}$ and $v_{g}$ may in fact be the same [2]. Because of the essential role of the energy velocity in describing the diffusive propagation of classical waves, it is crucial to develop a better understanding of $v_{e}$ by investigating its behavior over a wide range of frequencies, developing improved theoretical methods for accurately calculating its values, and reexamining its relationship to the group velocity.

In this Letter, we present a study of ultrasonic wave propagation through a strongly scattering medium in which we measure the energy velocity over an extended range of frequencies. This is accomplished by combining pulsed measurements of multiply scattered waves to determine $D$, and continuous-wave (cw) measurements of the thickness dependence of the absolute transmitted intensity to determine $l^{*}$. Insight into the behavior of the energy velocity is obtained by comparing our data with measurements of the group velocity, determined from ballistic pulse propagation in thin samples of the same strongly scattering material [13]. We find that the frequency dependence of the energy and group velocities is very similar; moreover, the two velocities are remarkably close in magnitude. Furthermore, the large dispersion that is observed in the energy velocity differs significantly from expectations based on theoretical calculations $[8,9]$ at comparable volume fractions of scatterers. We explain our experimental results using an effective medium model [14], based on a spectral function approach, that allows us to calculate $v_{e}$ quantitatively in the intermediate frequency regime, giving a simple and physically intuitive picture of the energy velocity in which we account explicitly for the scattering delay of a wave pulse [5,15]. In the forward direction, 
this scattering delay is accurately captured by this effective medium model of the group velocity, which gives an excellent description of the extremely slow values of $v_{g}$ measured in our experiments. We determine the energy velocity by accounting for the fact that the scattered waves experience an additional angle-dependent scattering delay, which is obtained directly within our model from the phase shifts given by the complex scattering amplitude for a single scatterer. The results of these calculations are in excellent agreement with our data; in addition, they provide the basis for calculating the frequency dependence of the diffusion coefficient, which is also in very good agreement with our measurements. Our model shows how the close relationship indicated by our data between the energy and group velocities can be explained in simple physical terms, and provides a unified picture of the transport velocities of classical waves in strongly scattering media.

The frequency-dependent diffusive transport of multiply scattered ultrasonic waves was measured in a strongly scattering medium consisting of monodisperse glass beads in water, randomly closely packed at a volume fraction of about $63 \%$. The ultrasonic wavelength was varied to span the normalized intermediate frequency range $1<k_{w} a<$ 9 , where $k_{w}$ is the ultrasonic wave vector in water and $a$ is the bead radius. The measurements were performed in a water tank using a plane-wave source, generated using a small-diameter immersion transducer, positioned far from the sample. A hydrophone, whose diameter was less than a wavelength, was used to detect the transmitted ultrasonic field in a single coherence area, or speckle spot, at the output face of the sample, enabling accurate measurements of the full transmitted ultrasonic wave form to be made [11]. Since we detect the full wave form, we can exploit the phase information in the acoustic field to separate the diffusively scattered component from the coherent ballistic component [13], allowing both types of propagation to be measured independently. An accurate ensemble average of the diffusive component was determined by scanning the hydrophone across the central portion of the sample face and averaging the square of the scattered field envelope over approximately 120 speckles.

The transport mean free path was determined from a cw measurement of the absolute transmission, which was obtained from the ratio of the transmitted to incident intensity in the middle of a very long pulse. Reliable values of $l^{*}$ were obtained by fitting the predictions of diffusion theory, in which absorption and boundary reflections are correctly included, to our data for the thickness dependence of the transmission [11]. Our results are shown by the solid symbols in Fig. 1; for comparison, we have also included data for the scattering mean free path $l_{s}$ (open symbols) obtained from the exponential attenuation $\exp \left(-L / l_{s}\right)$ of the ballistic intensity, measured using a short incident pulse [13]. At low frequencies $\left(2<k_{w} a<4\right)$, the values of the two mean free paths are indistinguishable within experimental error, while at higher frequencies, $l_{s}$ becomes

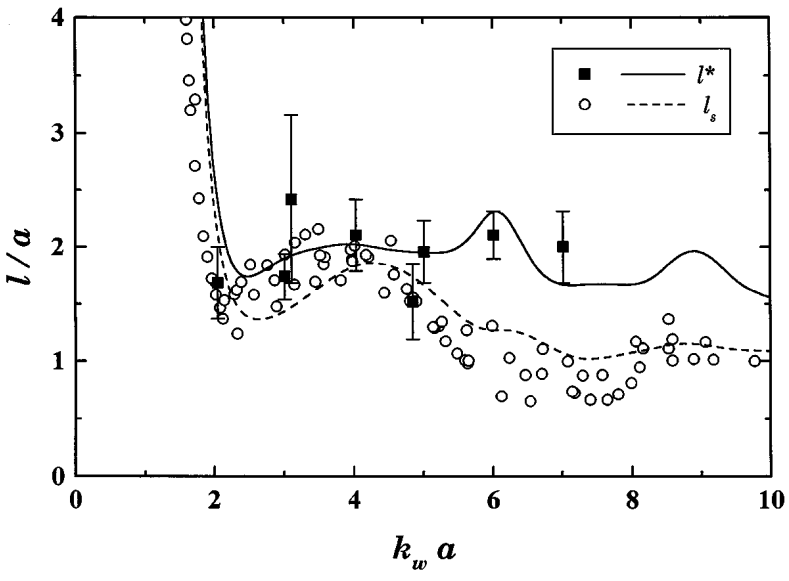

FIG. 1. Frequency dependence of the transport and scattering mean free paths (solid and open symbols, respectively), compared with the predictions of the effective medium model described in the text (solid and dashed curves).

appreciably smaller than $l^{*}$, the difference reflecting the growing anisotropy of the scattering. Furthermore, although there are pronounced minima in $l_{s}$ at $k_{w} a \approx 2$ and 7 , indicative of very strong resonant scattering, the transport mean free path exhibits less structure over this frequency range, and appears to be roughly independent of frequency in the strong scattering regime $k_{w} a \geq 2$.

The diffusion coefficient was determined in pulsed experiments by measuring the time dependence of the ensemble-averaged transmitted intensity of the scattered waves, and fitting the solution of the diffusion equation to these data, using appropriate boundary conditions to account for the reflectivity of the sample walls [11]. The measured frequency dependence of $D$ is shown by the solid symbols in Fig. 2; since we expect that $D$ scales as $l^{*}$, and thus $a$, we plot $D / a$ as a function of $k_{w} a$, enabling us to include data for different average bead radii, $a=0.25 \mathrm{~mm}$ (solid triangles) and $0.47 \mathrm{~mm}$ (solid squares). Some of these data were obtained using a point

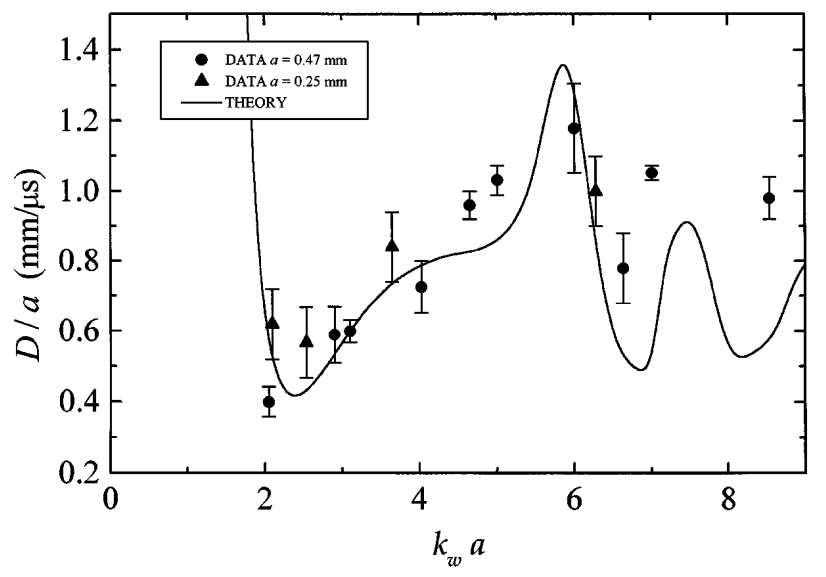

FIG. 2. Diffusion coefficient as a function of normalized frequency. 
source rather than a plane-wave source transducer; both sets of data are in excellent agreement with each other, confirming the robustness of the experiments and their analysis. The data exhibit pronounced structure as a function of frequency, varying by nearly a factor of 3 .

We use our data for $D$ and $l^{*}$ to determine the energy velocity $v_{e}=3 D / l^{*}$ and plot the results as a function of $k_{w} a$ using solid symbols in Fig. 3. For comparison, we also plot data for the group velocity of the same glassbead-in-water system, shown by the open symbols. The energy velocity is close in magnitude to the group velocity at all frequencies. In addition, both velocities exhibit considerable frequency dispersion, varying by about a factor of 3; this presumably results from the effects of scattering resonances, as the dips in the velocities occur at the same frequencies as the dips in the scattering mean free path. Near the first minimum in the group velocity, where the scattering mean free path becomes less than half the wavelength, the energy velocity is even a little slower than the already very slow group velocity; at the higher frequencies, the two velocities are indistinguishable within experimental error. Thus most of the dramatic slowing down of wave transport due to scattering resonances is captured by the group velocity that describes coherent pulse propagation, and the scattered waves experience a similar slowing down as they are scattered out of the forward direction. The origin of these very slow velocities can be described in terms of the propagation delay resulting from partial trapping of the wave energy by the resonant scatterers [4]; thus the small differences that are seen between the energy and group velocities for $2<k_{w} a<4$ reflect the additional delay experienced by a wave pulse as it is scattered through finite (nonzero) scattering angles.

To account quantitatively for this behavior, we use a model based on a spectral function approach, which overcomes a fundamental limitation of the traditional CPA and

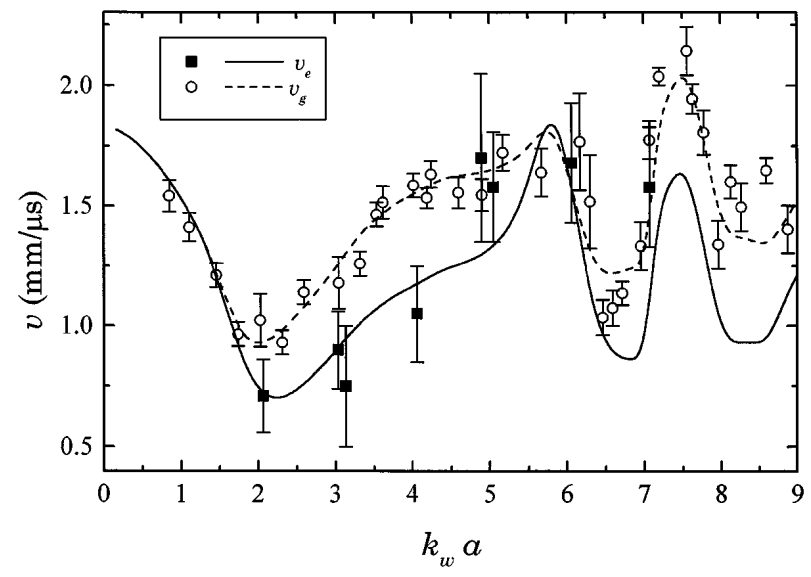

FIG. 3. Frequency dependence of the energy and group velocities (solid and open symbols, respectively), compared with the predictions of our model (solid and dashed curves). The theory curves in all of the figures are averaged over the small $5 \%$ variation in our bead size. allows wave propagation to be treated unambiguously in the presence of strong resonant scattering $[13,14]$. In this approach, the acoustic modes of the strongly scattering medium are identified by calculating the spectral function, given by the negative imaginary part of Green's function, $-\operatorname{Im} G(\omega, k)$. To evaluate the spectral function, we model a typical scatterer within the medium as an elastic sphere coated by a layer of water whose thickness is determined by the volume fraction, thus accounting for the strong geometric correlation that exists between the spheres and the surrounding fluid $[13,14]$. This coated sphere is embedded in a homogeneous medium whose wave speed is given by $v_{0}=\omega / k$ and, by solving the boundary value problem for the elastic wave equation, the spectral function is evaluated at all $\omega$ and $k$. Since the peaks in the density of states correspond to minima in the scattering, they delineate the propagating modes [16], determining the dispersion relation of the medium $\omega(k)$, from which both the phase and group velocities can be calculated directly [13]. The dashed line in Fig. 3 represents these theoretical predictions for $v_{g}$, and is seen to be in excellent agreement with the data, accurately capturing all the structure in the frequency dependence observed experimentally. Note that these calculations involve no adjustable parameters. We extend this approach to calculate the energy velocity by accounting for the additional scattering delay encountered by a wave pulse as it scatters from a coated sphere. At each scattering angle and frequency, we calculate the complex scattering amplitude $f\left(\omega, k, k^{\prime}\right)$ thus determining both the magnitude and the phase shift of each frequency component in the scattered pulse; the variation of these phase shifts with frequency results in a corresponding delay (or advance) of the scattered pulse envelope relative to the forward direction, which we determine directly by incorporating these phase and amplitude shifts into the Fourier components of an incident Gaussian pulse. In the simple physical picture of diffusive transport where the energy velocity is the average velocity of waves that have undergone many successive scatterings, with each scattering event altering the direction of propagation, $v_{e}$ is determined by taking the intensity-weighted angular average of these additional scattering delays, $\Delta t_{\text {ave }}$, giving to an excellent approximation $v_{e}=l^{*} /\left(l^{*} / v_{g}+\Delta t_{\text {ave }}\right)$, i.e., $v_{e}=v_{g} /\left(1+\Delta t_{\mathrm{ave}} v_{g} / l^{*}\right)$. Note that this expression for $v_{e}$ can be written as $v_{e}=v_{g} /\left(1+\delta_{m}\right)$, a form similar to that first proposed by the Amsterdam group [4], although, in our case, $v_{g}$ and $\delta_{m}$ are calculated in a renormalized effective medium which accounts for all the effects of the multiple scattering; here we explicitly identify $\delta_{m}=\Delta t_{\mathrm{ave}} v_{g} / l^{*}$, which is a small quantity in our approach. As shown by the solid line in Fig. 3, this calculation is in remarkably good agreement with the experimental data, correctly estimating the observed reduction of $v_{e}$ below $v_{g}$ for $2<k_{w} a<4$ while predicting that $v_{e}$ approaches $v_{g}$ for $5<k_{w} a<6$, which is also consistent with our data. Thus, by starting with an accurate 
calculation of the group velocity and then accounting for the additional scattering delay of a wave pulse, this theoretical model successfully describes the behavior of the energy velocity over an extended range of frequencies, revealing both the similarities and subtle differences between the energy and group velocities seen in our experiment.

The same theoretical model can also be used to calculate the scattering and the transport mean free paths. The scattering mean free path is obtained from the total scattering cross section of the coated sphere. Here, however, it underestimates the magnitude of the scattering because the neighborhood of a given particle is far from uniform and the single-sphere approximation does not adequately account for the additional random scattering from neighboring spheres [14]. We account for this effect empirically by a phenomenological, frequency independent, scaling parameter $p_{\sigma}$ determined through a fit to the data, yielding $p_{\sigma}=1.5$, which is consistent with exact multiple sphere scattering calculations [14]. As shown by the dashed curve in Fig. 2, we obtain excellent agreement with our experimental data, correctly accounting for the marked frequency dependence of $l_{s}$. The transport mean free path is determined using the same theoretical approach by integrating the square of the angle-dependent scattering amplitude, weighted by $(1-\cos \theta)$ where $\theta$ is the scattering angle, over all scattering angles. Since the scattering environment is the same, we use the same value for $p_{\sigma}$. We also obtain good agreement with the measured $l^{*}$, as shown by the solid curve in Fig. 2.

Since we have predicted the behavior of both $v_{e}$ and $l^{*}$, we can also determine the frequency dependence of the diffusion coefficient using $D=v_{e} l^{*} / 3$. The results are plotted as the solid line in Fig. 2; again the agreement between theory and experiment is very good, with the theory capturing all of the structure observed experimentally.

Our experimental results show convincingly that, at least for acoustic waves, there is considerable structure in the frequency dependence of the energy velocity at high volume fractions of scatterers. This structure is considerably greater than expected from previous calculations based on a CPA model [9], possibly indicating the shortcomings, noted above, of using the CPA approach in the intermediate frequency regime [12]; an additional deficiency of this CPA model is its unphysical prediction of an energy velocity that is substantially larger than both our measured and calculated values of the group velocity throughout most of the strong scattering regime investigated. By contrast, we find that the effect of the strong dispersion on the energy velocity is very similar to its effect on the group velocity, making it imperative to use our new approach to model their behavior theoretically. Underlying this behavior, however, is a simple physical picture. The coupling between the resonant scatterers leads to a renormalization of the scattering, so that the scattering from each sphere is weakened relative to the effective medium in which the spheres are embedded. This effect ensures that the group velocity remains well defined, and allows it to be accurately predicted by our model, which correctly describes the renormalized homogeneous medium that is sensed by the group velocity. The same mechanism also makes it possible to calculate the energy velocity quantitatively by extending the model to account for the angular dependence of the scattering delay. Furthermore, this renormalization of the scattering medium should increase with both the strength of the scattering and the volume fraction of scatterers, leading to the strong dispersion at high $\phi$ observed in our experiments. This physical picture has the appealing but generally unrecognized feature that the group and energy velocities are essentially the same [2], having a common origin in the velocity of propagation of a wave pulse, both for coherent, unscattered propagation, as well as for the propagation between scattering events in diffusive transport.

We thank NSERC, NATO, RGC (Grant No. HKUST685/96P), and NSF (Grant No. DMR9631279) for their support.

[1] Scattering and Localization of Classical Waves in Random Media, edited by P. Sheng (World Scientific, Singapore, 1990).

[2] P. Sheng, Introduction to Wave Scattering, Localization, and Mesoscopic Phenomena (Academic Press, San Diego, 1995).

[3] L. Brillouin, Wave Propagation and Group Velocity (Academic Press, New York, 1960).

[4] M. P. van Albada et al., Phys. Rev. Lett. 66, 3132 (1991); Phys. Rev. B 45, 12233 (1992); B. A. van Tiggelen and A. Lagendijk, Europhys. Lett. 23, 311 (1993).

[5] G. Cwilich and Y. Fu, Phys. Rev. B 46, 12015 (1992).

[6] E. Kogan and M. Kaveh, Phys. Rev. B 46, 10636 (1992).

[7] D. Livdan and A. A. Lisyansky, Phys. Rev. B 53, 14843 (1996).

[8] K. Busch and C. M. Soukoulis, Phys. Rev. Lett. 75, 3442 (1995); Phys. Rev. B 54, 893 (1996).

[9] M. Kafesaki and E. N. Economou, Europhys. Lett. 37, 7 (1997).

[10] A. Z. Genack et al., in Photonic Band Gaps and Localization, edited by C. M. Soukoulis (Plenum Press, New York, 1993), p. 23.

[11] J. H. Page et al., Phys. Rev. E 52, 3106 (1995).

[12] At intermediate frequencies where the wavelength is comparable with the size of the scatterers, it is well known that the self-energy acquires a wave vector dependence. As a result, the basis of the CPA approach, that the self-energy can be renormalized into a purely frequency dependent velocity, becomes invalid in any treatment involving resonant scattering.

[13] J. H. Page et al., Science 271, 634 (1996).

[14] X. D. Jing, P. Sheng, and M. Y. Zhou, Phys. Rev. Lett. 66, 1240 (1991); Phys. Rev. A 46, 6513 (1992); Physica (Amsterdam) 207A, 37 (1994).

[15] E. P. Wigner, Phys. Rev. 98, 145 (1955).

[16] These modes are really quasimodes, since they have finite decay lengths due to the strong scattering. 